\title{
Evolution and expression of the fructokinase gene family in Saccharum
}

Yihong Chen ${ }^{1,2}$, Qing Zhang ${ }^{1}$, Weichang Hư ${ }^{1}$, Xingtan Zhang ${ }^{1,3}$, Liming Wang ${ }^{1}$, Xiuting Hua ${ }^{1,2}$, Qingyi Yu $u^{1,4}$, Ray Ming ${ }^{1,5}$ and Jisen Zhang ${ }^{1,2,3^{*}}$

\begin{abstract}
Background: Sugarcane is an important sugar crop contributing up to about $80 \%$ of the world sugar production. Efforts to characterize the genes involved in sugar metabolism at the molecular level are growing since increasing sugar content is a major goal in the breeding of new sugarcane varieties. Fructokinases (FRK) are the main fructose phosphorylating enzymes with high substrate specificity and affinity.

Results: In this study, by combining comparative genomics approaches with BAC resources, seven fructokinase genes were identified in S. spontaneum. Phylogenetic analysis based on representative monocotyledon and dicotyledon plant species suggested that the FRK gene family is ancient and its evolutionary history can be traced in duplicated descending order: SSFRK4, SSFRK6/SSFRK7,SSFRK5, SSFRK3 and SSFRK1/SSFRK2. Among the close orthologs, the number and position of exons in FRKs were conserved; in contrast, the size of introns varied among the paralogous FRKs in Saccharum. Genomic constraints were analyzed within the gene alleles and between S. spontaneum and Sorghum bicolor, and gene expression analysis was performed under drought stress and with exogenous applications of plant hormones. FRK1, which was under strong functional constraint selection, was conserved among the gene allelic haplotypes, and displayed dominant expression levels among the gene families in the control conditions, suggesting that FRK1 plays a major role in the phosphorylation of fructose. FRK3 and FRK5 were dramatically induced under drought stress, and FRK5 was also found to increase its expression levels in the mature stage of Saccharum. Similarly, FRK3 and FRK5 were induced in response to drought stress in Saccharum. FRK2 and FRK7 displayed lower expression levels than the other FRK family members; FRK2 was under strong genomic selection constraints whereas FRK7 was under neutral selection. FRK7 may have become functionally redundant in Saccharum through pseudogenization. FRK4 and FRK6 shared the most similar expression pattern: FRK4 was revealed to have higher expression levels in mature tissues than in premature tissues of Saccharum, and FRK6 presented a slight increase under drought stress.
\end{abstract}

Conclusions: Our study presents a comprehensive genomic study of the entire FRK gene family in Saccharum, providing the foundations for approaches to characterize the molecular mechanism regulated by the SSFRK family in sugarcane.

Keywords: Fructokinase gene family, Saccharum, Gene expression, Gene evolution, Polyploidy

\section{Background}

Sugarcane (Saccharum spp.) is a large, perennial, tropical or subtropical crop. It is one of the world's most produced crops (FAOSTAT, 2015) contributing about 80\% of the world sugar production (FAOSTAT, 2010). Sugarcane also plays an increasingly important role in

\footnotetext{
* Correspondence: zjisen@126.com

${ }^{1}$ Center for Genomics and Biotechnology, Fujian Provincial Key Laboratory of Haixia Applied Plant Systems Biology, Haixia Institute of Science and Technology (HIST), Fujian Agriculture and Forestry University, Fuzhou 350002, China

${ }^{2}$ College of Life Sciences, Fujian Normal University, Fuzhou 350117, China

Full list of author information is available at the end of the article
}

the biofuel field accounting for $40 \%$ of ethanol production worldwide [1]. Sugarcane mature stalks contain about 9 to $18 \%$ sucrose [2]. The genus Saccharum includes two wild species, S. spontaneum and S. robustum, and one cultivar species $S$. officinarum; these three species are thought to be the founding species. Majority of both S. robustum and $S$. officinarum are octoploid, with a basic chromosome number $\mathrm{x}=8$, whereas $S$. spontaneum's chromosome number ranges from $2 n=36$ to 128 with the majority of basic chromosome number $\mathrm{x}=10$ [3-5]. S. officinarum is a high-glucose species with, and is thought to be derived from $S$. robustum as these two species have the same 
center of diversity in New Guinea [6]. Modern sugarcane cultivars are hybrids derived from the cross between $S$. officinarum and S. spontaneum, resulting in extreme allopolyploidy levels that can range from octoploidy $(x=8)$ to dodecaploidy $(x=12)$. Most of the basic molecular and genetic analyses remain inconclusive in sugarcane due to the lack of genomic information of this complex genome. Typically, expressed sequence tags (EST) resources are used for sugarcane gene and gene family discovery, and these were the sole resource prior to the release of sorghum genome $[7,8]$. Sorghum is the closest diploid species of Saccharum, and has a genome size about $760 \mathrm{Mb}$ which has been sequenced [9]. Comparative genomic studies demonstrated that sorghum has a small, diploid genome that contains fewer chromosomal rearrangements and shares a strong collinearity with sugarcane, thus providing the best reference model system to study sugarcane genomics [10-13]. It is possible to identify sugarcane genes by combining the BAC sequences and the available sorghum genome data.

The study of sugar metabolism in sugarcane is one of the most active areas of research. In plants, sucrose is cleaved into UDG-glucose and fructose by sucrose synthase (SUS), or can be cleaved into glucose and fructose by invertases for further sugar metabolism [14]. Fructokinases (FRK, EC 2.7.1.4) can phosphorylate free fructose with high substrate specificity and affinity [15]. Hence, the phosphorylation of fructose by FRKs is believed to be necessary for sucrose cleavage and sugar metabolism, both of which are essential for proper development of vascular tissue [16, 17]. To date, plant FRKs have been purified from several plants, such as Arabidopsis $[18,19]$, sugar beet [20], potato [21], tomato [22, 23], rice [24], soybean [25], maize [26], pea seeds [27]. Two isoforms of sugarcane fructokinases, FRK1 and FRK2 were purified from the culm of sugarcane [28]. Nevertheless, due to the complex sugarcane genetic background, identification of sugarcane FRK genes is still unavailable.

Understanding the molecular structure and evolution of a gene family is a key step towards the understanding of the physiological roles, metabolic mechanism and potential function of its members, the necessary groundwork for possible future transgenic studies. In this study, to characterize the gene evolution and possible functions of the FRK gene family in sugarcane, we performed gene family identification based on the combination of comparative genomics strategies and high genome coverage of bacterial artificial chromosomes (BACs) library resources, and investigated gene expression levels by RNA-seq and RT-qPCR. The analysis of this study mainly focuses on 1) the evolutionary relationship and gene structure of the FRK gene families, and 2) characterization of the gene expression patterns of the FRK gene family to predict function.

\section{Results}

Identification of seven FRK genes in S. spontaneum

Based on comparative genomics, seven well annotated sorghum FRK genes, referred to as SbFRKs, were identified (Table 1). These seven SbFRKs were located in chr01, chr03, chr07, chr09 and chr10 of sorghum genome. None of these seven was observed to have undergone tandem duplication. Using these well-annotated SbFRK genes as references to design probes (Table 1), 12 BAC sequences for FRKs were screened and sequenced from $S$. spontaneum. Further analysis revealed that the $12 \mathrm{~S}$. spontaneum sequences encoded seven FRKS referred to as SsFRK1-SsFRK7. In these 12 sequences, SsFRK1 had three allelic haplotypes, SsFRK2, SsFRK3, and SsFRK5 had two allelic haplotypes, SsFRK4, SsFRK6 and $S s F R K 7$ only had one allele. The allelic haplotypes of each FRK are indicated by an additional "-h1" to "-h3" at the end of gene name. Using the gene model sequences of the annotated FRK genes as queries, an in-house EST and Genbank database were extensively searched. The results showed that six of the SsFRKs had the corresponding ESTs in the Genbank database except SsFRK3 (Additional file 1).

The coding sequences of FRKs were translated into protein sequences. The alignment of SsFRKs revealed that SsFRKs harbors two conserved domains, pfkB1 [AG]-G-x $(0,1)-[G A P]-x-N-\{A G L S\}-[S T A]-x(2)-\{A\}-x-\{G\}-$ \{GNKA $\}-[G S]-x(9)-G$;and pfkB2:[DNSK]-[PSTV]-x-[SAG] (2)-[GD]-D-x(3)-[SAGV]-[AG]-[LIVMFYA]-[LIVMSTAP] (Fig. 1), demonstrating that these sequences belong to FRK family members. The molecular weights of the SsFRKs ranged between 34.7 and $66.2 \mathrm{kDa}$, and three FRKs (FRK3, FRK4, FRK5) had a larger size than the others (Table 2). Comparative analysis between sorghum and $S$. spontanenum were performed to investigate the divergence of SsFRKs/SbFRKs. The results showed that the SsFRKs have molecular weights that are similar to their orthologous FRK in sorghum, except for FRK6 and FRK7. Consistently, SsFRKs shared high identities ( $>=93 \%$ ) with their orthologous SbFRKs, well above those of FRK6 (77\%) and FRK7 (91\%). These results indicated that FRK6 and FRK7 had undergone stronger evolutionary dynamics after the split of Saccharum and Sorghum.

To analyze the divergence among the paralogous FRKs in $S$. spontaneum, an intercomparison of protein sequences among SsFRKs was performed (Additional file 2). Results showed that SsFRKs shared protein sequence similarities ranging between 25 and 64\%. Among the SsFRKs, SsFRK1 and SsFRK2 displayed the highest similarity, 64\%, whereas the other pairwise similarities were under $40 \%$, and most of the 14 of the analyzed gene pairs were excluded from the comparison results due to the high sequence divergence among the gene 
Table 1 Information of putative FRK genes in sorghum

\begin{tabular}{lllll}
\hline $\begin{array}{l}\text { Corresponding gene } \\
\text { name in sugarcane }\end{array}$ & Gene ID & Transcript ID & Protein ID & Location of the gene \\
\hline SSFRK1 & Sb03g042460 & XM_002458864.1 & XP_002458909.1 & NC_012872.1|:c69874432-69871609 chromosome 3 \\
SSFRK2 & Sb10g008280 & XM_002436715.1 & XP_002436760.1 & NC_012879.1|:8422480-8426810 chromosome 10 \\
SSFRK3 & Sb01g015030 & XM_002466795.1 & XP_002466840.1 & NC_012870.1|:c14441197-14437165 chromosome 1 \\
SSFRK4 & Sb01g046230 & XM_002468410.1 & XP_002468455.1 & NC_012870.1|:c69274783-69271917 chromosome 1 \\
SSFRK5 & Sb03g040010 & XM_002456599.1 & XP_002456644.1 & NC_012872.1|:67626538-67628943 chromosome 3 \\
SSFRK6 & Sb09g018040 & XM_002440925.1 & XP_002440970.1 & NC_012878.1|:c45125278-45122178 chromosome 9 \\
SSFRK7 & Sb07g027900 & XM_002445866.1 & XP_002445911.1 & NC_012876.1|:c62873182-62869987 chromosome 7 \\
\hline
\end{tabular}

families, suggesting that the $S s F R K$ is an ancient gene family with potentially high functional divergence.

\section{Comparative analysis of SsFRK allelic haplotypes}

Of the four SsFRKs with allelic haplotype sequences, three SsFRKs (SsFRK1, SsFRK2 and SsFRK5) shared very high similarity with identity higher than $99 \%$, and SsFRK3 was less constraint within its alleles of 93.33\% for genomic sequences and $95.58 \%$ for protein sequences. Similarly, gene structure comparisons within the allelic haplotype of the four genes also demonstrated that the allelic haplotype of three SsFRKS (SSFRK1, SsFRK2 and SsFRK5) were highly conserved, while SsFRK3 alleles had a deletion/insertion for their first intron (Fig. 2). Protein sequence alignment of the allelic haplotypes showed that SsFRK1, SsFRK2, SsFRK3 and SsFRK5 had 1, 3, 37 and 2 variant amino acids, respectively (Table 3 and Fig. 3). SsFRK3 presented great

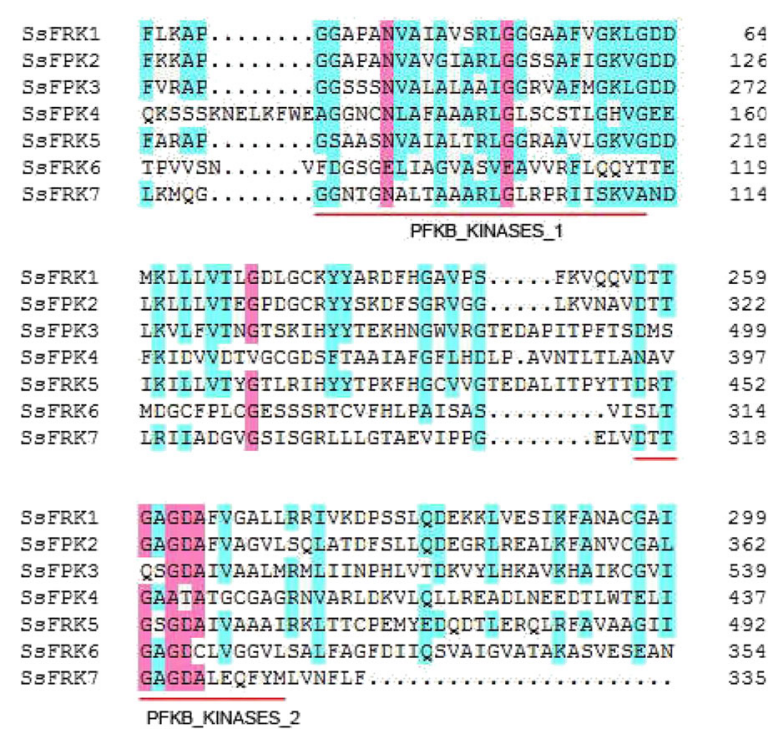

Fig. 1 Conserved domains of the SsFRKs gene family. Two conserved domains, pfkb1 and pfkb2, of FRKs were identified using BLASTp (http://blast.ncbi.nlm.nih.gov/Blast.cgi) and InterPro (http://www.ebi. ac.uk/interpro/scan.html) with protein sequences variation at the $\mathrm{N}$ terminal (Fig. 3). However, none of these variable amino acids located to the two pfrk domains, indicating functional conservation among these allelic haplotypes.

\section{The evolutionary function constraint in SsFRK families}

The evolutionary function constraint was evaluated for allelic haplotypes based on nonsynonymous to synonymous substitution ratio $(\mathrm{Ka} / \mathrm{Ks})$ (Fig. 4). The results revealed that SsFRK1,SsFRK2 and SsFRK5 were under strong purifying selection as the $K a / K s$ ratio was lower than 0.3 , while $F R K 3$ was approximately under neutral selection (pressure) with a $K a / K s$ ratio of 0.6. In addition, the $K a / K s$ ratio of pairwise of S.spontaneum sorghum showed that SsFRK4, SsFRK6, and SsFRK7 have a larger value above 0.6 . These results showed that different SsFRKs had undergone different evolutionary forces.

\section{Phylogenetic analysis of SsFRKs and other plant FRKs}

To analyze the phylogenetics of the FRK gene family, 71 FRKs were selected from eight representative plant species including four monocotyledons (sugarcane, sorghum, rice, and maize), and four dicotyledons (potato, Arabidopsis, grape, and tomato) and a phylogenetic tree was constructed using the ClustalX with NeighborJoining method (Fig. 5). The FRKs were phylogenetically distributed into six groups, referred to as group I group VI. Each of the groups contained FRK genes from both monocotyledons and dicotyledons, suggesting that the six gene groups originated before the monocot-dicot split. In group V, the dicot and monocot genes were separated into two subgroups, indicating that the genes in this group diverged from an evolutionary event after the common ancestor of dicots and monocots. Group I is the largest group, counting 32 FRKs including SsFRK1 and SsFRK2. In this group, genes were distributed into two subgroups, each of which could be further divides into two subgroups with one consisting of monocot specific genes, and the other containing both dicot and monocot genes, suggesting that gene expansion occurred in dicot species before the divergence of dicots and 
Table 2 Comparison of the characterization of the FRKs between sugarcane and sorghum

\begin{tabular}{|c|c|c|c|c|c|c|c|c|c|c|}
\hline \multicolumn{5}{|l|}{ Sorghum } & \multicolumn{5}{|l|}{ Sugarcane } & \multirow[b]{2}{*}{ Identity } \\
\hline Gene name & Protein size & Mw (kD) & Domains & Isoelectric point(pl) & Gene name & Protein size & $\mathrm{Mw}(\mathrm{kD})$ & Domains & Isoelectric point(pl) & \\
\hline SbFRK1 & 323 & 34.7 & PfkB & 5.00 & SSFRK1 & 323 & 34.7 & PfkB & 4.87 & $99 \%$ \\
\hline SbFRK2 & 388 & 41.2 & PfkB & 5.81 & SSFRK2 & 388 & 41.2 & PfkB & 5.56 & $97 \%$ \\
\hline SbFRK3 & 594 & 66.2 & PfkB & 7.23 & SSFRK3 & 583 & 65.0 & PfkB & 6.44 & $94 \%$ \\
\hline SbFRK4 & 488 & 52.2 & PfkB & 5.83 & SSFRK4 & 481 & 51.4 & PfkB & 6.09 & $93 \%$ \\
\hline SbFRK5 & 522 & 58.7 & PfkB & 6.36 & SSFRK5 & 524 & 58.9 & PfkB & 6.03 & $97 \%$ \\
\hline SbFRKG & 381 & 42.0 & PfkB & 5.33 & SsFRKG & 302 & 32.8 & PfkB & 5.43 & $91 \%$ \\
\hline SbFRK7 & 335 & 36.1 & PfkB & 9.14 & SSFRK7 & 364 & 38.2 & PfkB & 4.95 & $77 \%$ \\
\hline
\end{tabular}

monocots. Group II contained 11 FRKs including SsFRK3 and SsFRK5 from seven plant species except grape. Group III consists of 5 FRKs from 5 of the 8 plant species (excluding sugarcane, sorghum and potato), and it is likely that gene loss is a recent event (after the origin of Trib. Andropogoneae Dumort in monocots) that occurred in both of dicot and monocot plants. Both group IV and VI contain 8 FRKs with one from each the eight plant species, revealing that the genes in two groups shared an ancient ancestor before the divergence of dicots and monocots. Group V contained 7 FRKs from 7 of the 8 plant species (excluding potato), suggesting that a recent FRK gene loss event occurred after the split of potato and tomato in Solanum.

The FRKs of Chlamydomonas and yeast were used as the out group for the phylogenetic analysis of the origin of plant FRKs (Fig. 5). Group I and Group II were phylogenetically clustered together with an out group containing the FRKs of Chlamydomonas and yeast, whereas, group III, V and VI were clustered with different FRKs of Chlamydomonas and yeast. These results suggested that plant FRKs originated before the divergence of lower eukaryotes (such as Chlamydomonas and yeast) and land plants. Moreover, group VI were independently grouped together with a yeast FRK, indicating that a remote gene duplication event occurred (for the origin of O-II and O-III) before the split of yeast and land plants. In addition, group IV FRKs were separated from the other 5 groups and have not grouped with the lower eukaryotes gene, indicating that FRKs in this group are specific to land plants.

\section{Exon/Intron organization of the SsFRKs family and other plant FRKs}

In the examined plant species, the gene structures of $F R K$ were variable in both exon number and size. In group I, the exon number ranged from three to seven, while, the genes of each subgroup in this group contained similar exon numbers. In group II, the FRKs had exon numbers ranging from 2 to 6 , similarly genes in each of the subgroups had a similar exon number except for SSFRK3, which had 1 more exons. These results demonstrated that the exon/intron reorganization of FRKS is a recent evolutionary event that occurred after the split of dicots and moncots. Group III had two exons beside the corresponding genes of the grape in which the first exon was split in two yielding a total of four

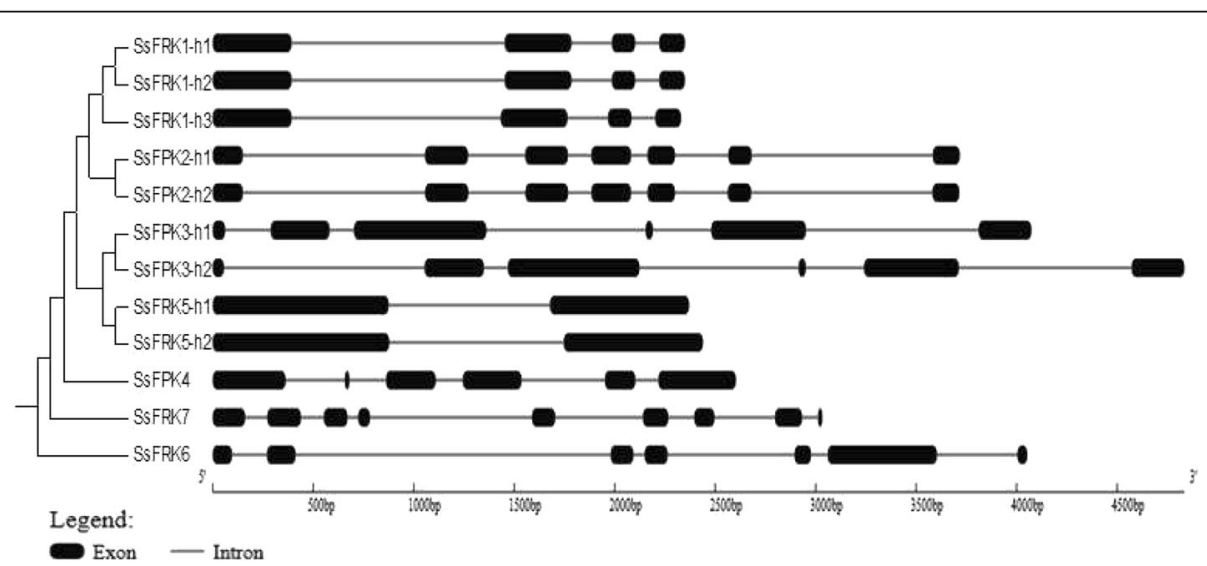

Fig. 2 Comparison of the allelic gene structure of SsFRKs. The allelic haplotypes of each FRK was indicated additional "-h1" to "-h3" at the end of gene name. Boxes represent exons 
Table 3 The variation of deduced amino acid sequences among allelic haplotypes within the four SsFRK

\begin{tabular}{lll}
\hline FRK & $\begin{array}{l}\text { No. of variations } \\
\text { (SsFRK-h1 vs } \\
\text { other haplotype) }\end{array}$ & Amino acid variations \\
\hline SSFRK1 & 1 & E160G \\
SSFRK2 & 3 & Q12R,16T,L190F \\
SSFRK3 & 37 & A2E,S3D,L4R,L6Y,P7Q,P8N,Q9E,L10H, \\
& & T11L,C12K,S13L,L14N,R15N,Y17L, \\
& & H18K,I20G,R21Q,G22-insertion,Q23L, \\
& & L24V,V30I,N34D,R45H,V47A,S48N,A58V, \\
& R75S,E94D,G95E,A100T,E101G,E110V, \\
& & E128-insertion,A177V,R319K,M4531,L565V
\end{tabular}

\begin{tabular}{lll} 
SSFRK4 & N/A & \\
SSFRK5 & 2 & T16P,106E-deletion \\
SSFRK6 & N/A & \\
SSFRK7 & N/A & \\
\hline
\end{tabular}

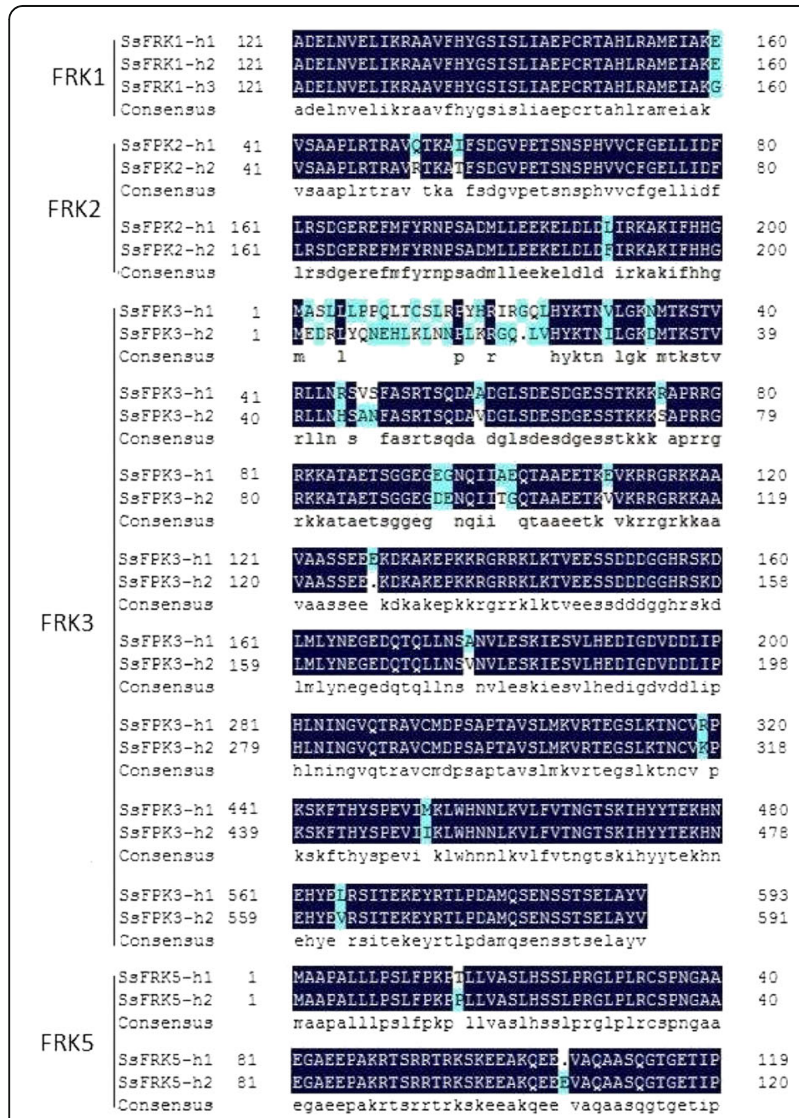

Fig. 3 Alignment of the amino acid sequences of SsFRK haplotypes. Amino acid sequences of haplotypes were aligned using the DNAMAN program. The difference between haplotypes is highlighted in blue exons and not three. In group IV, FRKs had five exons except SsFRK4 which harbors one additional exon originating from exonization in its corresponding first intron. In groups V and VI, FRKs harbored more and smaller exons than the genes in the other groups. Also, the gene sizes of the FRKs varied among the genes in these two groups due to intron expansion. Moreover, the FRKs in grape were found to have longer introns than other examined species, which is consistent with the results of whole genome analysis for grape genes [29].

The SsFRKs genes display great variation in exon numbers which range from 2 to 9 , with the introns aligning in accordance to the GT-AG rule for splicing sites (Fig. 2 and Fig. 5). Both SsFRK3 alleles contained one additional exon compared to its ortholog in sorghum, causing an exonization event in its third intron, indicating the gene restructured after the divergence of Saccharum and Sorghum and before whole genome duplication in S. spontaneum. Similarly, SsFRK4 harbored one additional exon compared to its ortholog in sorghum. These findings present evidence that the recent whole genome duplication of S. spontaneum provided the evolutionary forces for the restructuring of the SsFRK genes.

\section{Gene expression of SsFRKs among Saccharum species and Saccharum hybrid}

The gene identification makes it possible to investigate gene expression to evaluate the potential function of the gene families. We performed comparative transcriptome profiling among three Saccharum species and the Saccharum hybrid at different developmental stages of seedlings with different plant hormones and five different tissues from the mature leaf (mature and leaf roll) and stalks (mature, maturing and immature) by RNA-seq method. The Reads Per Kilobases per Million reads (RPKM) value of the examined genes were verified by q-RT-PCR in three tissue types from two Saccharum species, S. officinarum and S. spontaneum. The results were positively correlated with RPKM values (Additional file 3).

In all examined tissues, SsFRK1 was the most abundantly expressed gene among the SsFRK family, SsFRK7 presented the lowest expression levels (Fig. 6), and the remaining genes showed similar expression levels. These results suggested that $S s F R K 1$ was the dominant member of the gene family.

At the seedling stage, expression levels of FRK1s were higher in both S.officinarum and the Saccharum hybrid than in the other two species, which may be caused by that these two Saccharum plants had similar genetic background. Similar expression levels of SsFRKs were observed in the leaves and the stems, except the FRK3's expression that was higher in the stem than in leaves. At 


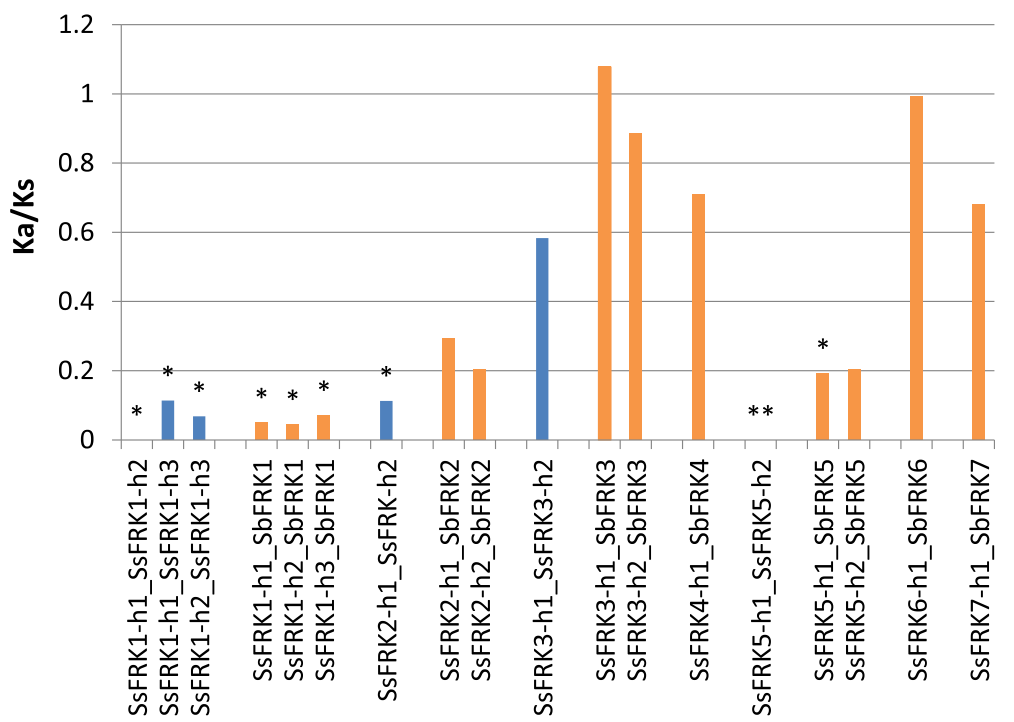

Fig. 4 The Ka/Ks of SsFRK haplotypes and SsFRK-SbFRK. The lower value of Ka/Ks is indicated by stars. The blue color indicates the Ka/Ks of gene pair allele comparisons, orange color indicates the Ka/Ks of orthologs genes between sorghum and S. spontaneum

the premature stage (7 months old), the expression of FRK3 was observed to decrease in comparison to seedling stages. In general, FRKs had higher expression levels in the mature period than in the premature period, suggesting that FRKs are involved in sugarcane sugar accumulation as mature plants contained higher sugar contents than the premature plants. The most abundantly expressed gene, FRK1, was observed to increase its expression levels in all analyzed leaf tissue including the leaf roll but decreased after the mature stage in some stem tissue (internode 3 of Saccharum hybrids and $S$. spontaneum, and internode 9 of S. officinarum), indicating that FRK1 is more active in the sugar metabolism of source tissues than in that of sink tissues. Similarly, FRK3 had higher expression levels in the source tissues than the sink tissues.

Polyethylene glycol (PEG) can be used to modify the osmotic potential of the nutrient solution culture and thus induce plant water deficit [30]. The accumulation of soluble sugars in response to drought stress has a positive correlation with the increase of leaf water contents [31-33]. In the leaves of seedling with PEG stress, the expression of FRK1 was suppressed in three of the examined Saccharum species (S. robustum, S. officinarum and hybrid cultivar (ROC-22) except S. spontaneum), where FRK1's expression was induced, FRK2's expression was not altered, and expression of the remaining five genes (FRK3, FKR4, FRK5, FRK6 and FRK7) increased. It was unexpected to observe that expression of FRK3 and FRK5 dramatically increased under PEG treatment, and had similar or higher mRNA levels than FRK1. These results strongly suggested that phosphorylation of fructose contributes to the sugar accumulation under PEG-induced drought stress, and both FRK3 and FKR5 were the main players in response to PEG stress.

Plant growth and development depend on the phytohormone-mediated regulation of gene expression. In this study, we analyzed the gene expression in the leaves of seedlings of Saccharum plants treated with abscisic acid (ABA), auxin (IAA), ethephon (Et), or gibberellins (GA). Gene expression levels were altered in response to ABA treatment, especially for SsFRK1, SsFRK3 and SsFRK5. Moreover, the gene expression of different FRKs varied in response to different ABA treatment times. For example, SsFRK1 showed a tendency to increase expression levels, whereas SsFRK5 decreased its expression level as the ABA treatment time was increased. No common shared gene expression change patterns could be identified for the FRK genes, suggesting that the FRKs are not genes directly regulated by $\mathrm{ABA}$. In contrast to the ABA treatment, the gene expression of $F R K s$ was not noticeably affected by the IAA treatment in Saccharum, except for FRK1, which had a more than five-fold increase in expression in the stems of $S$. officinarum after 24 h IAA treatment. In the leaves, SsFRK1 displayed a gradual decreasing trend at $24 \mathrm{~h}$, $48 \mathrm{~h}$ and $96 \mathrm{~h}$, but the trend was reversed in the stems. Under Et treatment, the expressions of FRK5 was suppressed in the majority of the examined tissues. Interestingly, FRK1 was suppressed in the leaves of the Saccharum hybrid and S. spontaneum for all three time points of Et treatment. As for the remaining 5 FRK genes, Et treatment did not generally cause a great variation in their expression levels. Similarly to the 


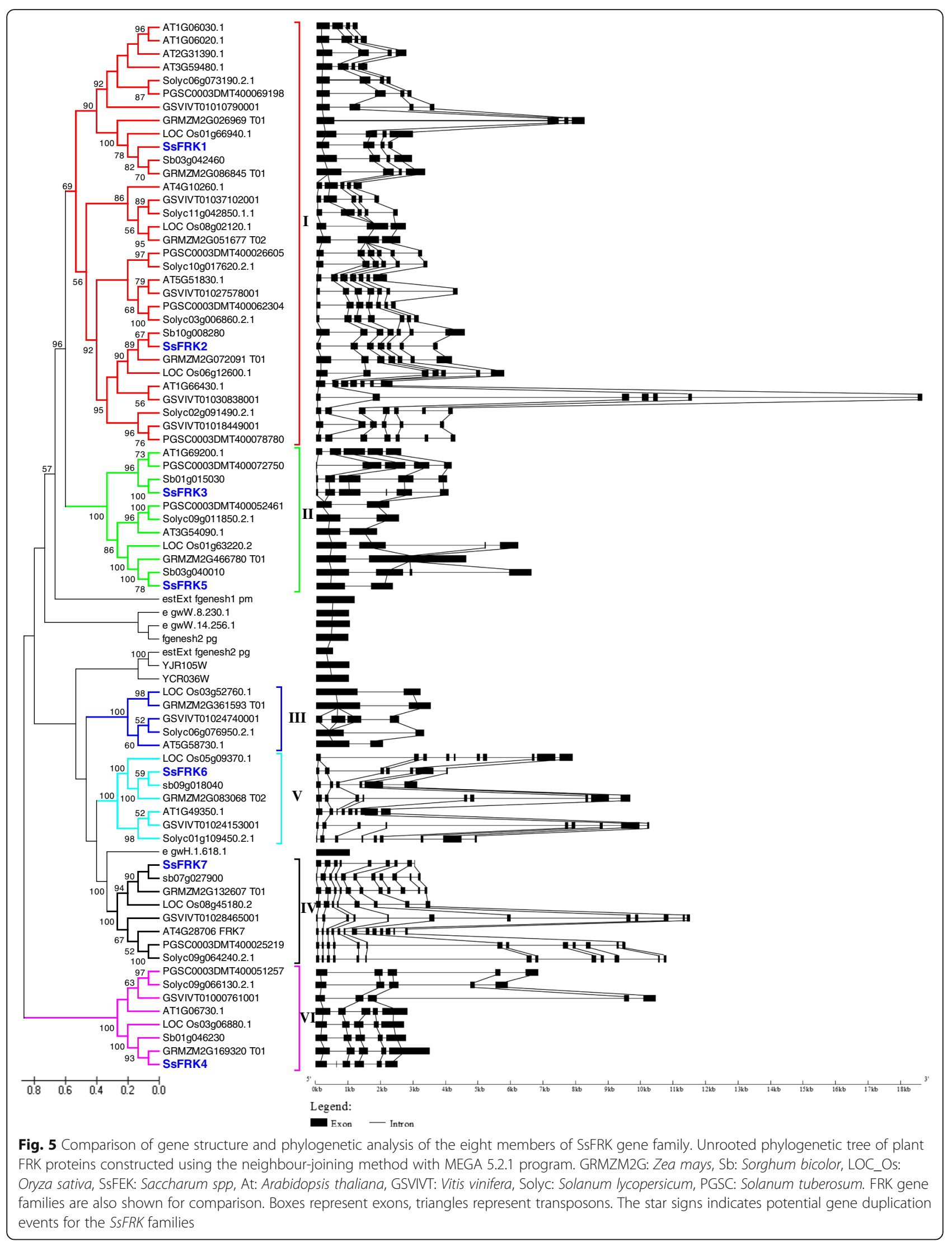



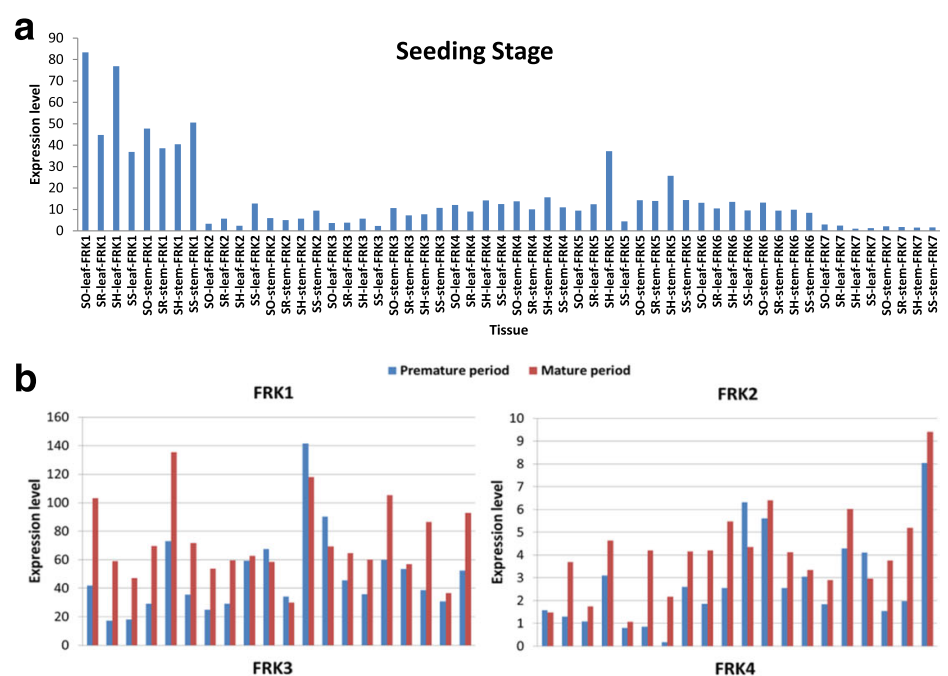

iod = Mature period
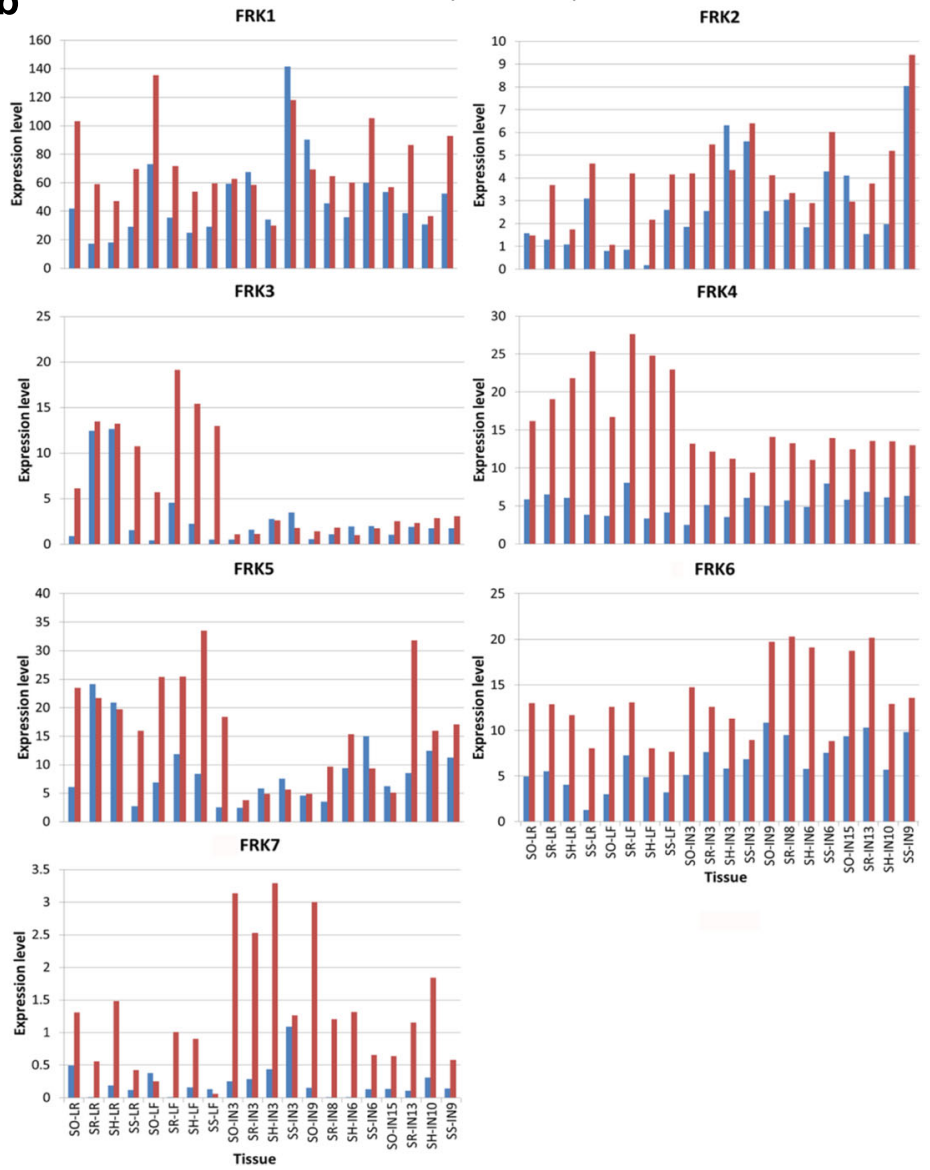

Fig. 6 a Expression levels of SsFRK gene family members at the seedling stage. SR: S. robustum Molokai6081; SS: S. spontaneum SES208; SO: S. officinarum LA Purple; SH: hybrid cultivar ROC-22. The gene expression level was based on RPKM value. $\mathbf{b}$ Expression levels of SsFRKs gene family members in pre-mature stage and mature stage tissues. SR: S. robustum Molokai6081; SS: S. spontaneum SES208; SO: S. officinarum LA Purple; SH: hybrid cultivar ROC-22; IN, internode; LR, leaf roll; LF, leaf. Internodes 3,9,15, internodes 3,9,15, internodes 3,8,13 and internodes 3, 6, 9 were from Saccharum officinarum (LA Purple), ROC-22, Saccharum robustum (Molokai6081) and Saccharum spontaneum (SES208), respectively. The gene expression level was based on RPKM value

treatment of GA of Saccharum plants, FRK5 expression was inhibited in the majority of examined tissues, and the transcript of FRK1 was inhibited in the leaf tissue in response to GA treatment at $48 \mathrm{~h}$ and $96 \mathrm{~h}$, but was induced in the stem tissues. These results demonstrated that FRKs were co-expressed under GA and Et treatments.

Cluster analysis of gene expression showed that the FRK family has a similar expression pattern in all experimental groups except for the GA treatment group, where $F R K 7$ s expression pattern resembled that of $F R K 2$ instead of FRK3 (Additional files 4, 5, 6, 7 and 8).

\section{Discussion}

In sugarcane, sugar metabolism is assumed to be one of the most important pathways in crop science due to its role of sugar accumulation. FRK is one of the main enzymes phosphorylating fructose to fructose 6-phosphate (F6P). Despite its essential role in fructose metabolism, FRKs are still poorly understood due to the complex 
sugarcane genome. Genomics and expressional studies are the key step for further gene function and molecular breeding studies. The available of sorghum genome [34], and the BAC of S. spontaneum make it possible to identify the FRK gene family members in Saccharum.

\section{Gene evolution in the FRK family}

In this study, seven FRKs were identified in the sorghum genome. Using sorghum FRKs as a reference, the same number of orthologous FRKs were identified in S. spontaneum. In comparison with previous studies on gene families in Saccharum, such as those on the phosphoenol pyruvate carboxylase gene, sucrose synthase [35], ATP-dependent phosphofructokinase [36], and sucrose transporters [37], SsFRKs presented higher divergence in their protein sequences (Additional file 2), indicating the FRKs are an ancient gene family. Phylogenetic analysis revealed that the FRK gene had undergone three rounds of gene duplication before the split of dicots and monocots (Fig. 5), further supporting the idea that FRKs are an ancient gene group.

Based on the phylogenetic analysis (Fig. 5), we can estimate the origin order of gene families. In the SsFRK family, the last common ancestor (LCA) of SsFRK was suggested to have undergone gene duplication four times. SsFRK4 was assumed to have originated from the first gene duplication, SsFRK6/SsFRK7 from the second, SsFRK3/ SsFRK5 from third, and the remaining two genes, SsFRK1 and SsFRK2, from the fourth. Therefore, the evolutionary history of SsFRKs could be sorted by age in duplicated descending order: SsFRK4, SsFRK6/ SsFRK7, SsFRK3/SsFRK5, and SsFRK1/SsFRK2. Of the four gene duplication events, the related SsFRK of the first two gene duplications were clustered together with different out groups of FRKs, suggesting these two times duplications occurred before the split of land plants and Chlamydomonas more than 10 billion years ago. Thus, FRKs are belong to an ancestral gene family that existed before the origin of land plants.

Sorghum is the closest diploid plant species of Saccharum, and comparison of protein sequences between orthologues of sorghum and S. spontaneum may provide clues to understand the gene evolution of recent whole genome duplications in Saccharum after the split of Sorghum and Saccharum. Based on the phylogenetic analysis, SsFRK1/SSFRK2 is suggested to be derived from the most recent duplication in this gene family, in addition to gene pairs of both SsFRK1 and SsFRK2 alleles were under strong selection constraints (Fig. 4), suggesting that these two genes were under functional constraint in S. spontaneum. Moreover, SsFRK1/SbFRK1 were more conserved in their protein sequence (99\%) than SsFRK2/SbFRK2 (97\%) (Table 2), Similarly, the gene structure and protein sequences were more conserved within the allelic haplotype of SsFRK1 than that of SsFRK2 (Fig. 2 and Table 3). Phylogenetic analysis may explain the differences of identities between these two recent duplication gene pairs in Saccharum. In the branch containing SsFRK1, Trib. Andropogoneae Dumort (sorghum and S. spontaneum) only has a single gene, while the other analyzed dicot plant species had at least two genes. But in the branch containing SsFRK2, all monocot plants only have one $F R K$. One of the FRKs in branch containing SsFRK1 was lost after the divergence of Trib. Andropogoneae Dumort in Gramineae, and thus SsFRK1 in Trib. Andropogoneae Dumort may replace the functional loss. Therefore, the FRK1 was under a stronger functional constraint than FRK2 in Trib. Andropogoneae Dumort.

FRK3 was present in Trib. Andropogoneae Dumort but was absent from both rice and maize, indicating that the function of FRK3 was replaceable in the Gramineae. $\mathrm{Ka}$ / $K s$ analysis suggesting that FRK3 had a lower selective constraint than FRK1, FRK4 and FRK5 (Fig. 4). It is possible that $F R K 3$ is functionally redundant in $S$. spontaneum. Alternatively, FRK3 may have been under positive selection, or demographic changes could have led to fixation of the most divergent allele in the S. spontaneum population.

SsFRK7 was the only SsFRK clustered with its orthologs in Chlamydomonas in a branch (VI) (Fig. 4), moreover, the SsFRK7/SbFRK7 had the lowest protein sequences similarity (77\%) among the seven orthologous gene pairs. Land plants and and Chlamydomonas diverged over 1 billion years ago [38]. This phylogenetic results thus suggested that $S s F R K 7$ had undergone a different evolution compared to other SsFRKs (Fig. 5). Ka/ Ks analysis revealed that SsFRK7 had lower selective constraint than other SsFRKs. Furthermore, SsFRK7 was distinct from the other SsFRKs for the protein sequence, and had the lowest gene expression levels among the SsFRKs. Therefore, the evolutionary mechanisms of SsFRK7 could be caused by its functional redundancy in $S$. spontaneum since the long term divergence after the LCA of SsFRK7 and the other SsFRKs.

\section{Gene expression and function in Saccharum plants}

Gene expression analysis could provide the first direct evidence to investigate gene function due to the challenges of engineering transgenic Saccharum. In this study, SsFRKs were found to be in the analyzed samples at widely different expression levels. SsFRK1 presented the highest gene expression levels among the SsFRKs, suggesting SsFRK1 is the key isoform involved in the phosphorylation of Fru to F6P, which may further explain the rationale that this gene was the most conserved among the SsFRK. However, under PEG-induced stress, the expression of FRK1 was suppressed except in $S$. 
spontaneum, which had expression levels similar to the control. Both FRK3 and FRK5 were induced dramatically by PEG treatment, especially $F R K 5$, which had higher gene expression levels than FRK1. Therefore, FRK1 was found to be the key isoform under normal conditions but not the key player for in the responses to PEG stress. Moreover, with the treatment of plant hormones at standard concentrations, our results showed that FRK1 had the predominant expression in the FRKS family (Figs. 6 and 7), suggesting that FRK1 is the key isoform for plant growth and development. Phylogenetic analysis showed that Solyc06g073190.2 was the closest orthologous gene of SsFRK2 in tomato; this gene is the main FRK in tomato plants [16] and was further found to be important for vascular development [16, 17]. Vascular development is the foundation for sugar accumulation, pointing to the importance of FRK1 in sugar accumulation in Sacchaurm in addition to sugar metabolism.

Both FRK2 and FRK7 had low expression levels in the examined tissues of the Saccharum, with FRK7 having the lowest expression levels. These two genes did not present clear expression variations under PEG stress and hormone treatments (Figs. 6 and 7), suggesting that these two genes do not play a main role in fructose metabolism. However, FRK2 was under strong selective constraint within the alleles and in comparison to sorghum, while FRK7 was under neutral selection. Therefore, we suspected that FRK2 had undergone a key functional division for the plant development, whereas FRK7 was functionally redundant in S. spontaneum. The close ortholog of the SsFRK2 gene in tomato (Solyc03g006860.2.1) is specifically expressed in the anthers during the late stages of pollen development and during pollen germination and has very low expression levels in the other tissues [39]. It is possible that FRK2 is involved in the reproductive system of Saccharum as this FRK has low expression in the leaf and stem tissues. Further analysis of FRKs gene expression in the development of pollen and anthers would be necessary to investigate the potential function of FRK2 in Saccharum plants.

$F R K 3$ and FRK5 were both dramatically induced by PEG treatment, therefore, the three FRKs including FRK1, FRK3 and FRK5 were predominant expression under drought stress (Fig. 7a). These results revealed that FRK3 and FRK5 played a main role in response to drought stress in Saccharum, and indicated that the phosphorylation of Glc to G6P and Fru to F6P were involved in response to drought stress in Saccharum. In another main sugar crop, sugar beet (Beta vulgaris) roots, FRK activity was observed to increase in response to wound stress [40]. In sunflower (Helianthus annuus), under drought stress, a plastid FRK is co-upregulated with other genes related to carbon metabolism [41]. In maize, FRK2 is upregulated in response to short- term salt stress [42]. In plants, FRKs are thought to be commonly involved in responses to abiotic stress. In contrast to FRK3, the expression levels of FRK5 were generally suppressed by Et and GA In young premature Saccharum plants plants, but increased in more mature tissues. GAs generally promote plant growth and greatly influence plant stature and organ size, while Et regulates stress-related responses and/or growth retardation. Therefore, FRK5 was suggested to play a role for plant growth in addition to stress tolerance response in Saccharum.

FRK4 and FRK6 had the closest expression profiles in most of the experiments from this study. Similarly to FRK5, the transcripts of FRK4 increased in the mature tissues of Saccharum (Fig. 6b) and under PEG stress (Fig. 7a), supporting the idea that $F R K s$ contribute to sugar accumulation and stress tolerance. Moreover, unlike FRK5, the gene expression of these two FRKs was not sensitive to the hormone treatments in the Saccharum plants. It was deduced that FRK4 and FRK6 were supposed to have different functional divisions to $F R K 5$ for plant growth and development. In addition, FRK6 only presented a slight increase in its expression levels under PEG-induced drought stress, indicating that FRK6 is not the major player in the response to drought stress in the FRK family in Saccharum.

Nevertheless, FRKs are considered to be the key enzymes for sugar metabolism in plants [16, 17]. In this study, we observed that FRKs generally have higher expression levels in mature tissues than in premature tissues (Fig. 6b), supporting the idea that FRK genes contribute to sugar accumulation as the mature tissues contained higher sugar content. However, in this study, there was no correlation between gene expression levels of FRKs and sugar content among the Saccharum species. Similarly, in our previous study for SUTs, no correlation was observed between gene expression levels of SUTs and sugar content in the Saccharum species [37]. These results suggest that the variation of sugar contents among the Saccharum species may not depend on the difference of gene expression of a small scales of sugar metabolism genes.

\section{Conclusions}

In this study, by combining comparative genomics approaches with BACs, we identified the fructokinase gene family consisting of seven members in S. spontaneum. We performed a comprehensive analysis of the evolutionary genesis, gene alleles, phylogenetic relationships, and gene expression patterns for the identified FRKs in Saccharum plants. The results suggest that the FRK gene family is an ancient gene family with ancestral LCA, and their evolution history could be sorted by age in $\mathrm{du}$ plicated descending order: SsFRK4, SsFRK6/SsFRK7, SsFRK3/ SsFRK5, and SsFRK1/SsFRK2. Moreover, 

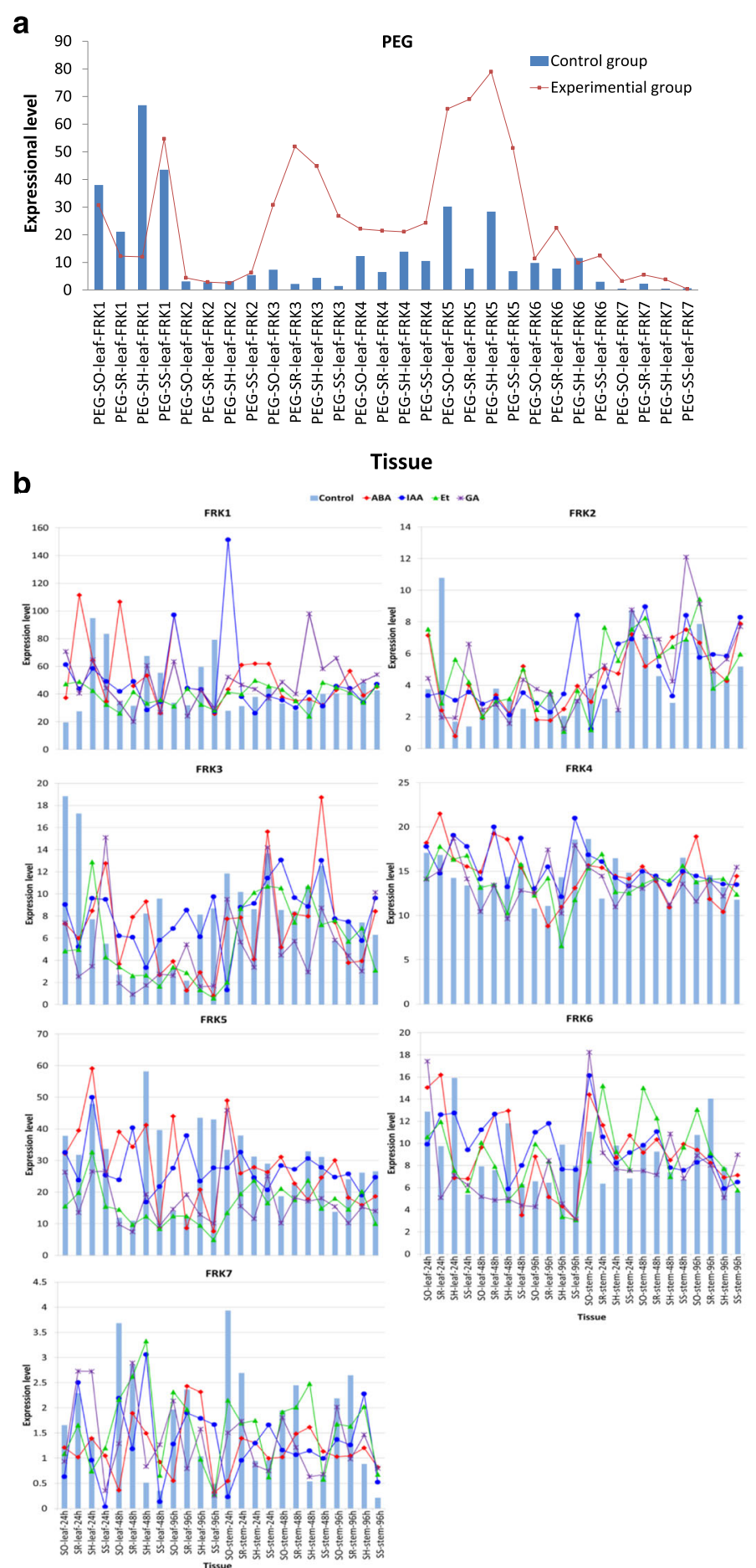

Fig. 7 a Expression levels of SsFRK gene family members under PEG treatment. SR: S. robustum Molokai6081; SS: S. spontaneum SES208; SO: S. officinarum LA Purple; SH: hybrid cultivar ROC-22; The gene expression level was based on RPKM value. $\mathbf{b}$ Expression levels of the SsFRK gene family members under hormone treatment (ABA, IAA, Et and GA). SR: S. robustum Molokai6081; SS: S. spontaneum SES208; SO: S. officinarum LA Purple; SH: hybrid cultivar ROC-22; IN: internode; LR: leaf roll; LF: leaf. Internnodes 3,9,15, internnodes 3,9,15, internodes 3,8,13 and internodes 3, 6, 9 were from Saccharum officinarum (LA Purple), ROC-22, Saccharum robustum (Molokai6081) and Saccharum spontaneum (SES208), respectively. The gene expression level was based on RPKM value 
individual FRKs were analyzed. FRK1 was under strong functional selection constraint and was conserved among the gene allelic haplotypes, in addition FRK1 displaying the highest expression levels among the other FRKs under normal condition, suggesting that FRK1 plays the leading role in phosphorylating Fru to F6P for plant development. FRK3 and FRK5 were identified as the key genes in response to drought stress. FRK5 was likely play a prominent role in plant growth in addition to stress tolerance response in Saccharum. FRK2 and $F R K 7$ had lower expression than the other gene members, whereas $F R K 2$ was under strong genomic selection constraint; FRK7 was under neutral selection. FRK2 may have undergone a functional division in Saccharum in agreement with the study of the SsFRK2 ortholog in tomato. FRK7 might be functionally redundant in Saccharum as a result of the process of pseudogenization. FRK4 and FRK6 had the closest expression pattern. FRK4 was revealed to have higher expression levels in mature tissues than in premature tissues of Saccharum, supporting the hypothesis that this gene contributes to the sugar accumulation in Saccharum plants. FRK6 presented slightly increase under drought stress. To investigate the function of these genes under stress, further experiments such as characterizing the spatio-temporal expression, enzyme activity assay, and targeted gene knock out technology like CRISPR-Cas9 system, would be necessary. This study may offer foundation work for the future study of the SsFRK gene family to characterize the physiological role and molecular mechanisms leading to sugar accumulation in sugarcane.

\section{Methods}

\section{Plant materials}

Two wild type Saccharum species, Molokai6081 ( $S$. robustum, SR $2 \mathrm{n}=8 \times=80$ ), and SES208 (S. spontaneum, SS, $2 \mathrm{n}=8 \times=64)$, one cultivated species, LA Purple (S. officinarum, SO, $2 \mathrm{n}=8 \times=80$ ), and one hybrid cultivar ROC-22 (SH $2 \mathrm{n}=8 \times=100-130$ ) were used in this study [43]. Plants were grown in the field on the campus of Fujian Agricultural and Forestry University (Fuzhou, China) in the February of 2015. Tissue samples from leaf roll, leaf, top internode (i.e., internode number 3), maturing internode (i.e., internode number 9 for 'LA Purple and Roc-22, internode number 8 for Molokai6081, and internode number 6 for SES208) and mature internode (i.e., internode number 15 for 'LA Purple' and Roc-22), internode number 13 for Molokai6081, internode number 9 for SES208) were collected from premature 7-month-old sugarcane plants and 11-month-old mature sugarcane plants from different branches of the same individuals (as replicates). Internodes were numbered from the top, as previously described [44], and the corresponding internode number for the different Saccharum species, due to the variation in number of stems, was also established according to the previously described approach [45].

The plants for PEG and hormone treatment were grown in a growth chamber at $30{ }^{\circ} \mathrm{C}, 70 \% \mathrm{RH}$, and a $14 \mathrm{~h}: 10 \mathrm{~h}$ L:D photoperiod. Seedlings were treated with PEG6000 (30\%) for $48 \mathrm{~h}$, and the leaf tissue was collected for RNA isolation. Seedlings were treated with gibberellin (GA,200 $\mu \mathrm{M})$, abscisic acid(ABA, $200 \mu \mathrm{M})$, indole-3-acetic acid(IAA, $200 \mu \mathrm{M}$ ), or ethephon (Et, $200 \mu \mathrm{M}$ ) for 24, 48, and $96 \mathrm{~h}$. Stem and leaf tissues from the seedlings of the four sugarcane species were collected from 35-day-old plants. Harvested tissue was immediately frozen in liquid nitrogen and stored at $-80{ }^{\circ} \mathrm{C}$ prior to RNA isolation.

\section{Database search for the FRKs gene family}

Due to the lack of reference information for whole FRK gene families in plant, we used the keyword "fructokinase" to search the model plant Arabidopsis genome database (TAIR 10 release, http://www.Arabidopsis.org/), and the rice genome database (http://rice.plantbiolo gy.msu.edu/, release 5.0). These sequences were then used to search FRKs from the Phytozome (http://phyto zome.jgi.doe.gov/pz/portal.html) and GenBank for monocots - sorghum (Sorghum bicolor), maize (Zea mays), dicots - potato (Solanum tuberosum), grape (Vitis vinifera) and tomato (Solanum lycopersicum). The sequences containing the pfkB domain with matches achieved similarity scores of 50.0, probability scores $>50.0$ and e-value $<10-4$, and were collected.

\section{Identification of $F R K$ families from a BAC library}

A BAC library was constructed for the haploid genome of S. spontaneum SES208, Ap85-441 $(2 \mathrm{n}=4 \times=$ 32 ), and contained 38,400 clones with an average insert size of $100 \mathrm{~kb}$, resulting in $6 \times$ coverage of the whole genome [36].

As described by $\mathrm{Yu}$ et al. [45], BAC library screening was performed using the probe amplified with the primers shown in Additional file 9. The positive BAC clones were verified by PCR with the same primers and then sequenced according to the Sanger method by Takara (Dalian, China). Different haplotypes were selected. BAC DNAs were isolated using the PhasePrep ${ }^{\mathrm{mm}}$ TMBAC DNA kit (Sigma-Aldrich, NA0100-1KT), and the insert size of BAC clones was estimated by comparison with standard size markers using CHEF gel electrophoresis. The DNA of individual BAC clones was prepared with unique barcode using the DNA Library Prep Kit (NEB \#E7645). The DNA-seq libraries were then pooled and sequenced with $150 \mathrm{bp}$, pair-end reads on Illumina HiSeq2500 at the Center for Genomics and Biotechnology in Fujian Agriculture 
and Forestry University. The BAC sequences were then assembled using SPAdes Genome Assembler v. 3.1.1(http://bioinf.spbau.ru/en/spades).

\section{Sequence analysis and phylogenetic tree}

The BAC assembled contigs were annotated by DNA subway (http://dnasubway.iplantcollaborative.org/) with Sorghum and sugarcane ESTs from GenBank as references, the CDS sequences of the $F R K$ genes were then translated into protein sequences by the EXPASytranslate tool (http://web.expasy.org/translate/). The putatively conserved domains of FRK proteins were detected by using BLASTp (http://blast.ncbi.nlm.nih.gov/ Blast.cgi) and InterPro (http://www.ebi.ac.uk/interpro/ scan.html). The isoelectric point and relative molecular mass of the proteins were predicted using ExPASy (http://web.expasy.org/compute_pi/). The exon-intron structures for the FRK genes were graphed using the online tool GSDS (http://gsds.cbi.pku.edu.cn/).

The amino acid sequences of $F R K$ family members in 4 monocotyledons (Zea mays, Sorghum bicolor, Oryza sativa, and Saccharum spontaneum), and 4 dicotyledons (Arabidopsis thaliana, Solanum lycopersicum, Solanum tuberosum and Vitis vinifera) were used for phylogenetic tree analysis. The phylogenetic trees were constructed with the MEGA5.2.1 program, ClustalW alignment using default parameters.

We calculated pairwise distances between FRK alleles of S. spontaneum and their orthologs from Sorghum at synonymous sites (Ks) and non-synonymous sites (Ka) by using DnaSP (version 5.10.1 http://www.ub.edu/ dnasp/) with default parameters. The FRK orthologs between S. spontaneum and sorghum were identified based on phylogenetic analysis and the protein sequence identities. The FRK genes of S. spontaneum and sorghum which phylogenetic distributed together and shared higher sequence similarity were considered to be the orthologs genes.

\section{Analysis of the co-expression profiling of fructokinases in Saccharum based on RNA-seq}

$5 \mu \mathrm{g}$ total RNA of each sample were used for the construction of cDNA libraries. The cDNA libraries were prepared using Illumina ${ }^{-}$TruSeq $^{\mathrm{mm}}$ RNA Sample Preparation Kit (RS-122-2001(2), Illumina) according to the manufacturer's protocol. The RNA-seq libraries were pooled and sequenced with 100 single reads on Illumina HiSeq2500 at the Center for Genomics and Biotechnology at the Fujian Agriculture and Forestry University.

Raw data were aligned to reference gene models using NOVOALIGN (http://www.novocraft.com/). NOVOALIGN reports multiple alignments for each read, however, the program discards alignments if the posterior alignment probability of the best alignment is less than 0.7 (http://www.novocraft.com/documentation/novoalign-2 /novoalign-user-guide/novoalign-command-options/report ing-multiple-alignments-per-read/). Mappable reads were counted using htseq-count program (http://www-hu ber.embl.de/users/anders/HTSeq/doc/count.html) with union mode. To identify differentially expressed genes (DEGs), we applied edgeR package to calculate Reads Per Kilobase of exon model per Million mapped reads(RPKM) value and fold change (FC) for each gene [46]. Genes with $|\log 2 \mathrm{FC}>2|, \mathrm{FDR}<0.05$ were considered as DEGs.

\section{Validation of RPKM values for FRK genes using qRT-PCR}

RNA $(\leq 1 \mu \mathrm{g})$ from each sample was reverse-transcribed to cDNA using the Reverse Transcriptase Kit (Takara) in a $20 \mu \mathrm{l}$ reaction volume with $1 \mu \mathrm{l}$ of random primers and $1 \mu \mathrm{l}$ of mixed poly-dT primers (18-23 nt). The cDNA was diluted 1:7 in water for further qRT-PCR experiments.

The expression levels of $F R K$ genes were validated using qRT-PCR in partial tissues of four sugarcane species. Gene-specific primer pairs (Additional file 9) were designed using the online PrimerQuest tool at Integrated DNA Technologies (IDT) (http://www.idtdna.com/Pri merquest/Home/Index). Real-time qPCR were run on Multicolor Real-Time PCR Detection System (Bio-Rad). The real time PCR reaction program was $95{ }^{\circ} \mathrm{C}$ for 30s, 40 cycles at $95{ }^{\circ} \mathrm{C}$ for $5 \mathrm{~s}$, followed by $60{ }^{\circ} \mathrm{C}$ for $30 \mathrm{~s}$, and PCR specificity was confirmed using a heat dissociation protocol from 65 to 95 C following the final cycle of the PCR. The glyceraldehyde-3-phosphate dehydrogenase gene (GAPDH) and Eukaryotic elongation factor 1a (eEF1a) were selected as internal standards for normalization [47], and three replicates were run for each sample. The relative expression levels for each $F R K$ gene in different tissues of three sugarcane species were calculated using the $2^{-\Delta \Delta C t}$ method [48].

\section{Additional files}

Additional file 1: BLAST results for ESTs of SsFRK in the NCBI database. (DOC 29 kb)

Additional file 2: Amino acid sequence pairwise comparisons (\% similarity) between FRK gene family members in sugarcane. (DOC 31 kb) Additional file 3: Comparison of gene expression levels of SSFRK gene family members by qRT-PCR and RNA-Seq. PM: premature; SES: S. spontaneum SES208; LA: S. officinarum LA Purple; IN:internode; LF, leaf. Using the data made a correlation analysis. (PPTX $119 \mathrm{~kb}$ )

Additional file 4: Heatmap of the expression levels of SSFRK gene family members in pre-mature stage and mature stage tissues. SR: S. robustum Molokai6081; SS: S. spontaneum SES208; SO: S. officinarum LA Purple; SH: hybrid cultivar ROC-22; IN: internode; LR: leaf roll; LF: leaf. Internnodes 3,9,15, internnodes 3,9,15, internodes 3,8,13 and internodes 3, 6, 9 were from Saccharum officinarum (LA Purple), ROC-22, Saccharum robustum (Molokai6081) and Saccharum spontaneum (SES208), respectively. (PPTX $156 \mathrm{~kb}$ ) 
Additional file 5: Heatmap of the expression levels of SSFRK gene family members under ABA treatment. SR: S. robustum Molokai6081; SS: S. spontaneum SES208; SO: S. officinarum LA Purple; SH: hybrid cultivar ROC22; IN: internode; LR: leaf roll; LF: leaf. Internnodes 3,9,15, internnodes 3,9,15, internodes 3,8,13 and internodes 3, 6, 9 were from Saccharum officinarum (LA Purple), ROC-22, Saccharum robustum (Molokai6081) and Saccharum spontaneum (SES208), respectively. (PPTX $205 \mathrm{~kb}$ )

Additional file 6: Heatmap of the expression level of SSFRK gene family members under IAA treatment. SR: S. robustum Molokai6081; SS: S. spontaneum SES208; SO: S. officinarum LA Purple; SH: hybrid cultivar ROC-22; IN: internode; LR: leaf roll; LF: leaf. Internnodes 3,9,15, Internnodes $3,9,15$, internodes 3,8,13 and internodes 3, 6, 9 were from Saccharum officinarum (LA Purple), ROC-22, Saccharum robustum (Molokai6081) and Saccharum spontaneum (SES208), respectively. (PPTX 194 kb)

Additional file 7: Heatmap of the expression levels of SSFRK gene family members under Et treatment. SR: S. robustum Molokai6081; SS: S. spontaneum SES208; SO: S. officinarum LA Purple; SH: hybrid cultivar ROC-22;IN: internode; LR: leaf roll; LF: leaf. Internnodes 3,9,15, internnodes 3,9,15, internodes 3,8,13 and internodes 3, 6, 9 were from Saccharum officinarum (LA Purple), ROC-22, Saccharum robustum (Molokai6081) and Saccharum spontaneum (SES208), respectively. (PPTX $193 \mathrm{~kb})$

Additional file 8: Heatmap of the expression levels of SsFRK gene family members under GA treatment. SR: S. robustum Molokai6081; SS: S. spontaneum SES208; SO: S. officinarum LA Purple; SH: hybrid cultivar ROC-22; IN: internode; LR: leaf roll; LF: leaf. Internnodes 3,9,15, Internnodes 3,9,15, internodes 3,8,13 and internodes 3, 6, 9 were from Saccharum officinarum (LA Purple), ROC-22, Saccharum robustum (Molokai6081) and Saccharum spontaneum (SES208), respectively. (PPTX $195 \mathrm{~kb}$ )

Additional file 9: The primers for qRT-PCR verification of five SSFRK in four Saccharum species. (DOC $29 \mathrm{~kb}$ )

\section{Abbreviations}

ABA: Abscisic acid; ATP: Adenosine triphosphate; BACs: Bacterial artificial chromosomes; EST: Expressed sequence tags; Et: Ethephon; F6P: Fructose 6-phosphate; FRK: Fructokinases; Fru: Fructose; GA: Gibberellins; IAA: Auxin; LCA: The last common ancestor; Mya: Million years ago; PEG: Polyethylene glycol; RPKM: Reads Per Kilobases per Millionread

\section{Acknowledgments}

We thank I. Lavagi for critical reading and language editing. We also thank two anonymous reviewers for their constructive comments and suggestions, which helped us to improve the manuscript.

\section{Funding}

This project was supported by grants from the 863 program (2013AA102604), and NSFC (31201260) and the funding from the Fujian Agriculture and Forestry University.

\section{Availability of data and materials}

The 14 sequences of SSFRKs(allele haplotype)were deposited into Genbank (accession numbers: KX430841-KX430852).

\section{Authors' contributions}

YC and JZ conceived and designed the experiments. YC,QZ, WH, and JZ performed the experiments. YC,QZ,WH,LW, XH, and JZ performed data analysis. QY and RM provided materials and references. YC and JZ drafted the manuscript. YC,XZ and JZ revised the manuscript. All authors read and approved the final manuscript.

\section{Competing interests}

The authors declare that the research was conducted in the absence of any commercial or financial relationships that could be construed as a potential conflict of interest.

\section{Consent for publication}

All the authors hereby consent to publish this work in BMC Genomics.

Ethics approval and consent to participate

Not applicable.

\section{Author details}

${ }^{1}$ Center for Genomics and Biotechnology, Fujian Provincial Key Laboratory of Haixia Applied Plant Systems Biology, Haixia Institute of Science and Technology (HIST), Fujian Agriculture and Forestry University, Fuzhou 350002, China. ${ }^{2}$ College of Life Sciences, Fujian Normal University, Fuzhou 350117, China. ${ }^{3}$ Key Laboratory of Ministry of Education for Genetics, Breeding and Multiple Utilization of Crops, Fujian Agriculture and Forestry University, Fuzhou 350002, China. ${ }^{4}$ Texas A\&M AgriLife Research, Department of Plant Pathology and Microbiology, Texas A\&M University System, Dallas, TX 75252, USA. ${ }^{5}$ Department of Plant Biology, University of Illinois at Urbana-Champaign, Urbana, IL 61801, USA.

Received: 28 July 2016 Accepted: 2 February 2017 Published online: 21 February 2017

\section{References}

1. Lam E, Shine J, Da Silva J, Lawton M, Bonos S, Calvino M, Carrer H, SilvaFilho MC, Glynn N, Helsel Z. Improving sugarcane for biofuel: engineering for an even better feedstock. GCB Bioenergy. 2009;1(3):251-5.

2. Tai $P$, Miller J. Germplasm diversity among four sugarcane species for sugar composition. Crop Sci. 2002:42(3):958-64.

3. D'Hont A, Ison D, Alix K, Roux C, Glaszmann JC. Determination of basic chromosome numbers in the genus Saccharum by physical mapping of ribosomal RNA genes. Genome. 1998;41(2):221-5.

4. Ha S, Moore PH, Heinz D, Kato S, Ohmido N, Fukui K. Quantitative chromosome map of the polyploid Saccharum spontaneum by multicolor fluorescence in situ hybridization and imaging methods. Plant Mol Biol. 1999;39(6):1165-73.

5. Irvine JE. Saccharum species as horticultural classes. Theor Appl Genet. 1999;98:186-94

6. Daniels J, Roach BT. Taxonomy and evolution. Amsterdam: Sugarcane improvement through breeding Elsevier; 1987. p. 7.

7. Borecký J, Nogueira FT, De Oliveira KA, Maia IG, Vercesi AE, Arruda P. The plant energy-dissipating mitochondrial systems: depicting the genomic structure and the expression profiles of the gene families of uncoupling protein and alternative oxidase in monocots and dicots. J Exp Bot. 2006; 57(4):849-64.

8. Sculaccio SA, Napolitano HB, Beltramini LM, Oliva G, Carrilho E, Thiemann $\mathrm{OH}$. Sugarcane phosphoribosyl pyrophosphate synthetase: molecular characterization of a phosphate-independent PRS. Plant Mol Biol Report. 2008;26(4):301-15.

9. Paterson AH, Bowers JE, Bruggmann R, Dubchak I, Grimwood J, Gundlach H, Haberer G, Hellsten U, Mitros T, Poliakov A, et al. The Sorghum bicolor genome and the diversification of grasses. Nature. 2009;457(7229):551-6.

10. Jannoo N, Grivet L, Chantret N, Garsmeur O, Glaszmann JC, Arruda P, D'Hont A. Orthologous comparison in a gene-rich region among grasses reveals stability in the sugarcane polyploid genome. Plant J. 2007;50(4):574-85.

11. Wang J, Roe B, Macmil S, Yu Q, Murray JE, Tang H, Chen C, Najar F, Wiley G, Bowers J. Microcollinearity between autopolyploid sugarcane and diploid sorghum genomes. BMC Genomics. 2010;11(1):261.

12. Dufour P, Deu M, Grivet L, D'hont A, Paulet F, Bouet A, Lanaud C, Glaszmann J, Hamon P. Construction of a composite sorghum genome map and comparison with sugarcane, a related complex polyploid. Theor Appl Genet. 1997; 94(3-4):409-18

13. Ming R, Liu S-C, Lin Y-R, Da Silva J, Wilson W, Braga D, Van Deynze A, Wenslaff T, Wu K, Moore P. Detailed alignment of Saccharum and Sorghum chromosomes: comparative organization of closely related diploid and polyploid genomes. Genetics. 1998;150(4):1663-82.

14. Dennis D, Blakeley S. Carbohydrate metabolism. Biochem Mol Biol Plants. 2000;2000:630-75

15. Renz A, Stitt M. Substrate specificity and product inhibition of different forms of fructokinases and hexokinases in developing potato tubers. Planta. 1993;190(2):166-75.

16. German MA, Dai N, Matsevitz T, Hanael R, Petreikov M, Bernstein N, loffe M, Shahak Y, Schaffer AA, Granot D. Suppression of fructokinase encoded by LeFRK2 in tomato stem inhibits growth and causes wilting of young leaves. Plant J. 2003;34(6):837-46.

17. Damari-Weissler H, Rachamilevitch S, Aloni R, German MA, Cohen S, Zwieniecki MA, Holbrook NM, Granot D. LeFRK2 is required for phloem and xylem differentiation and the transport of both sugar and water. Planta. 2009; 230(4):795-805 
18. Kaplan CP, Tugal HB, Baker A. Isolation of a cDNA encoding an Arabidopsis galactokinase by functional expression in yeast. Plant Mol Biol. 1997;34(3):497-506.

19. Alexandrov NN, Troukhan ME, Brover W, Tatarinova T, Flavell RB, Feldmann KA. Features of Arabidopsis genes and genome discovered using full-length cDNAs. Plant Mol Biol. 2006;60(1):69-85.

20. Chaubron F, Harris N, Ross HA, Davies HV. Partial purification and characterization of fructokinase from developing taproots of sugar beet (Beta vulgaris). Plant Sci. 1995;110(2):181-6.

21. Taylor MA, Ross HA, Gardner A, Davies HV. Characterisation of a cDNA encoding fructokinase from potato (Solanum tuberosum L.). J Plant Physiol. 1995;145(3):253-6.

22. Kanayama Y, Dai N, Granot D, Petreikov M, Schaffer A, Bennett AB. Divergent fructokinase genes are differentially expressed in tomato. Plant Physiol. 1997;113(4):1379-84.

23. German MA, Asher I, Petreikov M, Dai N, Schaffer AA, Granot D. Cloning, expression and characterization of LeFRK3, the fourth tomato (Lycopersicon esculentum Mill.) gene encoding fructokinase. Plant Sci. 2004;166(2):285-91.

24. Jiang $H$, Dian W, Liu F, Wu P. Isolation and characterization of two fructokinase cDNA clones from rice. Phytochemistry. 2003;62(1):47-52.

25. Kuo TM, Doehlert DC, Crawford CG. Sugar metabolism in germinating soybean seeds: evidence for the sorbitol pathway in soybean axes. Plant Physiol. 1990;93(4):1514-20.

26. Sr Z, Nichols SE, Dong JG. Cloning and characterization of two fructokinases from maize. Plant Sci. 2003;165(5):1051-8.

27. Copeland L, Stone SR, Turner JF. Kinetic studies of fructokinase I of pea seeds. Arch Biochem Biophys. 1984;233(2):748-60.

28. Hoepfner SW, Botha FC. Expression of fructokinase isoforms in the sugarcane culm. Plant Physiol Biochem. 2003;41(8):741-7.

29. Jaillon O, Aury JM, Noel B, Policriti A, Clepet C, Casagrande A, Choisne N, Aubourg S, Vitulo N, Jubin C, et al. The grapevine genome sequence suggests ancestral hexaploidization in major angiosperm phyla. Nature. 2007;449(7161):463-7.

30. Lagerwerff JV, Ogata G, Eagle HE. Control of osmotic pressure of culture solutions with polyethylene glycol. Science. 1961;133(3463):1486-7.

31. Parida AK, Dagaonkar VS, Phalak MS, Umalkar G, Aurangabadkar LP. Alterations in photosynthetic pigments, protein and osmotic components in cotton genotypes subjected to short-term drought stress followed by recovery. Plant Biotechnol Rep. 2007:1(1):37-48.

32. Irigoyen J, Einerich D, Sánchez-Díaz M. Water stress induced changes in concentrations of proline and total soluble sugars in nodulated alfalfa (Medicago sativd) plants. Physiol Plant. 1992;84(1):55-60.

33. Wu Q-S, Xia R-X. Arbuscular mycorrhizal fungi influence growth, osmotic adjustment and photosynthesis of citrus under well-watered and water stress conditions. J Plant Physiol. 2006;163(4):417-25.

34. Bowers JE, Miguel AA, Rochelle A, Jennifer AA, Robert TB, Gene AB, Ryan WB, Amy HC, Thomas ME, James CE, Heather EE, Valorie HG, Kristen LH, Cassie LS, Santhosh K, Gmerice KL, Cornelia L, Barry SM, Shelley LM, Joana MM, Lisa KN, Graham AN, Chike CN, Rosana NO, Cynthia AP, Elizabeth AR, Carl JR, Sean PR, Keimun AS, Carol AS, Haibao T, Rod AW, Andrew HP. Comparative physical mapping links conservation of microsynteny to chromosome structure and recombination in grasses. Proc. Natl Acad. Sci. USA. 2005;102(37):13206-211.

35. Besnard G, Pincon G, D'Hont A, Hoarau JY, Cadet F, Offmann B. Characterisation of the phosphoenolpyruvate carboxylase gene family in sugarcane (Saccharum spp.). Theor Appl Genet. 2003;107(3):470-8.

36. Zhu L, Zhang J, Chen Y, Pan H, Ming R. Identification and genes expression analysis of ATP-dependent phosphofructokinase family members among three Saccharum species. Funct Plant Biol. 2013;40(4):369-78.

37. Zhang Q, Hu W, Zhu F, Wang L, Yu Q, Ming R, Zhang J. Structure, phylogeny, allelic haplotypes and expression of sucrose transporter gene families in Saccharum. BMC Genomics. 2016;17:88.

38. Merchant SS, Prochnik SE, Vallon O, Harris EH, Karpowicz SJ, Witman GB, Terry A, Salamov A, Fritzlaylin LK, Maréchaldrouard L. The Chlamydomonas Genome Reveals the Evolution of Key Animal and Plant Functions. Science. 2007:318(5848):245-50

39. David-Schwartz R, Weintraub L, Vidavski R, Zemach H, Murakhovsky L, Swartzberg D, Granot D. The SIFRK4 promoter is active only during late stages of pollen and anther development. Plant Sci. 2013;199:61-70.

40. Klotz KL, Finger FL, Anderson MD. Wounding increases glycolytic but not soluble sucrolytic activities in stored sugarbeet root. Postharvest Biol Tec. 2006;41(1):48-55.

41. Fulda S, Mikkat S, Stegmann H, Horn R. Physiology and proteomics of drought stress acclimation in sunflower (Helianthus annuus L.). Plant Biol. 2011;13(4):632-42.
42. Zorb C, Schmitt S, Muhling KH. Proteomic changes in maize roots after shortterm adjustment to saline growth conditions. Proteomics. 2010;10(24):4441-9.

43. Zhu DC, Liu L, Gao F, Li Q, Zhang B. Comparison of closed reduction and expert tibial nailing with open reduction and plate and screw fixation in the treatment of two segmental tibial fractures. Chin J Traumatol. 2015;18(4):219-22.

44. Moore PH. Anatomy and morphology. Sugarcane Improv Breed. 1987;1987: 85-142.

45. Cho MH, Lim H, Shin DH, Jeon JS, Bhoo SH, Park YI, Hahn TR. Role of the plastidic glucose translocator in the export of starch degradation products from the chloroplasts in Arabidopsis thaliana. New Phytol. 2011;190(1):101-12.

46. Mortazavi A, Williams BA, McCue K, Schaeffer L, Wold B. Mapping and quantifying mammalian transcriptomes by RNA-Seq. Nat Methods. 2008;5(7):621-8.

47. Ling H, Wu Q, Guo J, Xu L, Que Y. Comprehensive selection of reference genes for gene expression normalization in sugarcane by real time quantitative rt-PCR. PLoS One. 2014:9(5):e97469.

48. Pfaffl MW. A new mathematical model for relative quantification in real-time RT-PCR. Nucleic Acids Res. 2001:29(9):e45.

\section{Submit your next manuscript to BioMed Central and we will help you at every step:}

- We accept pre-submission inquiries

- Our selector tool helps you to find the most relevant journal

- We provide round the clock customer support

- Convenient online submission

- Thorough peer review

- Inclusion in PubMed and all major indexing services

- Maximum visibility for your research

Submit your manuscript at www.biomedcentral.com/submit
Biomed Central 